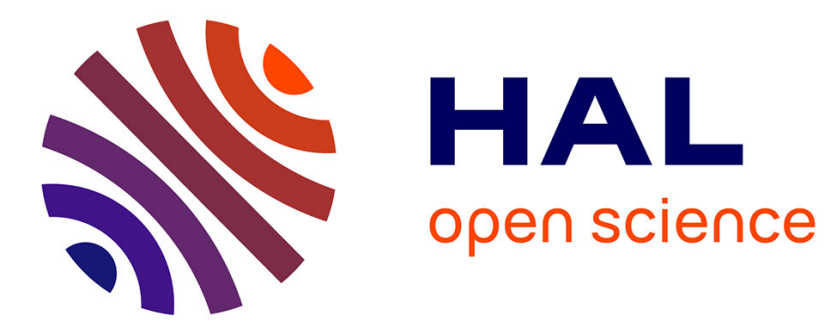

\title{
Expérience d'optique
}

Emeric Dubois

\section{- To cite this version:}

Emeric Dubois. Expérience d'optique. J. Phys. Theor. Appl., 1881, 10 (1), pp.448-451. 10.1051/jphystap:0188100100044800 . jpa-00237843

\section{HAL Id: jpa-00237843 https://hal.science/jpa-00237843}

Submitted on 1 Jan 1881

HAL is a multi-disciplinary open access archive for the deposit and dissemination of scientific research documents, whether they are published or not. The documents may come from teaching and research institutions in France or abroad, or from public or private research centers.
L'archive ouverte pluridisciplinaire HAL, est destinée au dépôt et à la diffusion de documents scientifiques de niveau recherche, publiés ou non, émanant des établissements d'enseignement et de recherche français ou étrangers, des laboratoires publics ou privés. 


\section{EXPÉRIENGE D'OPTIQUE;}

PtR M. E. DUBOIS, Professeur au lycée d'Amiens.

On sait que la lumière blanche est complètement éteinte par son passage à travers deux verres colorés de teintes pures et différentes, par exemple un verre rouge et un verre vert. Pour le démontrer dans les cours publics, on prendra deux plaques de bois semblables à celles qui servent à supporter les vues ou les dessins que l'on veut projeter, et, après y avoir pratiqué des ouvertures circulaires égales, on encartera dans l'une un verre vert, dans l'autre un verre rouge, se coupant à angle droit au centre du cercle, comme

Fig. 1.

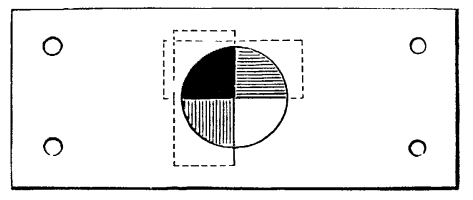

l'indique la figure, lorsque les deux plaques auront été superposées et fixées l'une à l'autre par quatre vis. Le cercle obtenu en projection est divisé en quatre secteurs, le premier blanc, le deuxième rouge, le troisième vert, le quatrième noir. Ce dernier correspond au secteur dans lequel les deux verres sont superposés.

Cette expérience est inverse de celle qui consiste, à l'aide de deux appareils de projection, à superposer sur un écran blanc un faisceau rouge et un faisceau vert, ce qui donne du blanc, le rouge et le vert étant complémentaires.

OBERBECK. - On the friction in free surfaces of liquids (Sur le frottement à la surface libre des liquides); Phil. Magazine, $5^{\circ}$ série, t. XI, p. 132 ; г88.

Il semble résulter des expériences de M. Plateau que la viscosité des liquides n'est pas la mème à leur intérieur qu'à leur surface 
libre. Ce physicien trouve, en faisant osciller une aiguille aimantée à l'intérieur, puis à la surface d'un liquide, que l'eau, les dissolutions salines, la glycérine, les solutions d'albumine et de savon sont plus visqueuses à la surface qu'à l'intérieur, tandis que le contraire a lieu pour l'alcool, la térébenthine, l'éther, le sulfure de carbone. M. Marangoni attribue, au contraire, ces différences à la capillarité et à la présence de corps étrangers à la surface des liquides.

Pour résoudre la question, l'auteur emploie une croiv de laiton suspendue par deux fils de platine. Les bras sont constitués par des vis le long desquelles peuvent se déplacer des poids quand on veut changer la durée d'oscillation. Un miroir fixé à cette croix et une lunette permettent d'étudier les oscillations. La branche inférieure porte une plaque mince ou un cylindre de laiton qui plonge plus ou moins profondément dans le liquide placé au-dessous et oscille à son intérieur en tournant autour d'un axe vertical. Une vis micrométrique permet d'élever ou d'abaisser l'ensemble de l'appareil.

La plaque était d'abord entièrement plongée dans le liquide, puis on l'élevait de façon à placer sa face supérieure dans le plan de la surface libre, et enfin au-dessus de ce plan. On observait la durée des oscillations et la diminution progressive de leur amplitude. Le liquide était contenu dans un vase étroit, car, dans un vase large, le disque, oscillant, entraine dans son mouvement le liquide qui l'entoure, ce qui diminue beaucoup l'influence du frottement, comme l'ont montré les expériences exécutées avec des vases de différentes largeurs. Dans les plus grands, la réaction du liquide masque presque entièrement l'influence de la situation dı corps oscillant et celle de l'addition d'un liquide étranger à la surface du premier liquide, tandis que ces influences apparaissent nettement avec un vase étroit.

Comme on observe toujours des oscillations de faible amplitude, on peut considérer la résistance du liquide comme proportionnelle à la vitesse angulaire de l'apparcil. On a ainsi pour l'équation du mouvement

$$
\frac{d^{2} \Phi}{d t^{2}}+2 a \frac{d \Phi}{d t}+b \Phi=0
$$


\{5o OBERBECK. - FROTTEMENT A LA SURFACE DES LIQUIDES. et $\Phi$ peut s'exprimer par la formule

$$
\Phi=\Phi_{0} e^{-\frac{i t}{\mathbf{T}}} \cos \left(2 \pi \frac{t}{\mathbf{T}}\right)
$$

Le coefficient $\frac{\lambda}{\mathrm{T}}=a$ peut être considéré comme mesurant la décroissance progressive de l'amplitude, et par suite le frottement du liquide sur le solide mobile. D'après l'hypothèse précédente, cette quantilé doit être indépendante de l'amplitude, ainsi que la durée d'oscillation. En réalité, la durée d'oscillation reste, en effet, constante dans une même expérience; mais, quand la face supérieure du disque s'élève au-dessus du liquide, une action capillaire entre en jeu sur ses bords et ralentit un peu l'oscillation. Quant à la décroissance, elle diminue lentement quand l'amplitude s'abaisse; il suffit de considérer ses valeurs moyennes.

Les expériences ont conduit aux résultats suivants :

$\mathbf{1}^{\circ}$ Dans tous les liquides observés, la résistance croît quand le disque approche de la surface libre. Ce résultat paraît dù à ce que les molécules liquides, écartées de leur position par le disque, ne peuvent plus facilement s'échapper en remontant, à cause du voisinage de la surface. Il est possible aussi que l'évaporation de la surface abaisse la température des couches voisines et change la résistance.

$2^{\circ}$ Quand la face supérieure du disque atteint et dépasse la surface libre, les liquides se partagent en plusieurs groupes, comme l'avait indiqué M. Plateau. Les uns, comme l'eau et les dissolutions salines, présentent une augmentation de résistance considérable; d'autres, comme l'alcool, le sulfure de carbone, l'essence de térébenthine, présentent une diminution de résistance; les mélanges d'eau et d'alcool affectent des allures qui dépendent de leurs proportions. Une faible addition d'alcool modifie beaucoup les propriétés de l'eau. L'accroissement de résistance à la surface de l'eau et des solutions salines ne tient pas à la présence de corps étrangers à leur surface. La filtration du liquide et son agitation, qui change l'état de la surface, n'exercent qu'une influence assez faible sur la résistance observée. La diminution de résistance à la surface de l'alcool et des autres liquides de la seconde série est d'autre part trop faible pour qu'on en puisse tirer des conclusions bien certaines. 
NiCHOLS ET THEELER. - DILATATION DES DISSOLUTIONS, ETC. $45 \mathrm{I}$

$3^{\circ}$ Pour les grandes profondeurs, le sulfure de carbone offre la plus faible résistance; celles de l'eau et de l'alcool diffèrent peu l'une de l'autre; celle de l'essence de térébenthine est beaucoup plus grande. L'addition des sels augmente la résistance de l'eau. Les mélanges d'eau et d'alcool résistent plus que ces deux liquides séparés.

Foussereau. 